PROCEEDINGS OF THE

AMERICAN MATHEMATICAL SOCIETY

Volume 132, Number 12, Pages 3607-3614

S 0002-9939(04)07435-0

Article electronically published on July 20, 2004

\title{
INFINITE SYSTEMS OF LINEAR EQUATIONS FOR REAL ANALYTIC FUNCTIONS
}

\author{
P. DOMAŃSKI AND D. VOGT \\ (Communicated by N. Tomczak-Jaegermann)
}

\begin{abstract}
We study the problem when an infinite system of linear functional equations

$$
\mu_{n}(f)=b_{n} \quad \text { for } n \in \mathbb{N}
$$

has a real analytic solution $f$ on $\omega \subseteq \mathbb{R}^{d}$ for every right-hand side $\left(b_{n}\right)_{n \in \mathbb{N}} \subseteq \mathbb{C}$ and give a complete characterization of such sequences of analytic functionals $\left(\mu_{n}\right)$. We also show that every open set $\omega \subseteq \mathbb{R}^{d}$ has a complex neighbourhood $\Omega \subseteq \mathbb{C}^{d}$ such that the positive answer is equivalent to the positive answer for the analogous question with solutions holomorphic on $\Omega$.
\end{abstract}

\section{INTRODUCTION}

One of the first classical examples of an infinite system of linear equations is related to the so-called moment problem solved by Hausdorff. It is the question of finding a Borel measure $\mu$ on $[0,1]$ such that a given sequence of reals $\left(b_{n}\right)_{n \in \mathbb{N}}$ is the sequence of moments of $\mu$ (i.e., $\int_{0}^{1} t^{n} d \mu(t)=b_{n}$ for $n \in \mathbb{N}$ ). More generally, we can look for a functional $f \in X^{\prime}$ such that for the given sequence of scalars $\left(b_{n}\right)$ and of vectors $\left(x_{n}\right)$ belonging to a locally convex (Banach) space $X$ the following holds:

$$
f\left(x_{n}\right)=b_{n} \quad \text { for } n \in \mathbb{N} \text {. }
$$

First solutions for spaces $X=C[0,1]$ or $L_{p}[0,1]$ are due to F. Riesz (1909) and the functional analytic approach to the problem is contained in the famous book of Banach [1, Ch. IV §7, §8]; see also [23, pp. 106-107].

Later on, Eidelheit, a colleague of Banach, characterized in all Fréchet spaces what are now called Eidelheit sequences [9] (see [17, Th. 26.27], [12, II.38.6]). Let $X$ be a locally convex space. We call a sequence $\left(\mu_{n}\right)_{n} \in \mathbb{N}$ of continuous linear functionals on $X$ an Eidelheit sequence on $X$ if for every sequence of scalars $\left(b_{n}\right)_{n} \in \mathbb{N}$ there is $f \in X$ satisfying

$$
\mu_{n}(f)=b_{n} \quad \text { for } n \in \mathbb{N} .
$$

The notion of Eidelheit sequences has been extensively studied in Fréchet spaces later on; see [9], [18], [20], 21] and [22], compare also [17, §26].

Received by the editors January 28, 2003 and, in revised form, May 22, 2003 and July 9, 2003. 2000 Mathematics Subject Classification. Primary 46E10; Secondary 46A13, 26E05, 46F15.

Key words and phrases. Space of real analytic functions, analytic functionals, interpolation of real analytic functions, Eidelheit sequence. 
We will prove a completely analogous result for the highly non-metrizable space of real analytic functions $\mathscr{A}(\omega)$ on an arbitrary open set $\omega \subseteq \mathbb{R}^{d}$ (Theorem [2.2). We show that for every open set $\omega \subseteq \mathbb{R}^{d}$ there is a domain of holomorphy $\Omega \subseteq \mathbb{C}^{d}$, $\Omega \cap \mathbb{R}^{d}=\omega$, such that every Eidelheit sequence on $\mathscr{A}(\omega)$ is automatically Eidelheit on the space $H(\Omega)$ of holomorphic functions on $\Omega$ (Theorem 2.2). The main tool in the proof is given by the so-called distinguished sets, which might be of independent interest (see Lemma 1.1 and Lemma 1.3).

Let $\left(f_{1, n}\right)_{n} \in \mathbb{N},\left(f_{2, n}\right)_{n} \in \mathbb{N}$ be two Eidelheit sequences on $\mathscr{A}\left(\omega_{1}\right)$ and $\mathscr{A}\left(\omega_{2}\right)$, respectively. We consider the question if there is a continuous linear map (=operator) $T: \mathscr{A}\left(\omega_{1}\right) \rightarrow \mathscr{A}\left(\omega_{2}\right)$ such that $f_{1, n}=f_{2, n} \circ T$ for every $n \in \mathbb{N}$ (compare an analogous problem on Fréchet spaces due to Mityagin [18] and its solution in [21]). It turns out that for every $\left(f_{1, n}\right)_{n \in \mathbb{N}}$ there is $\left(f_{2, n}\right)_{n} \in \mathbb{N}$ without such factorization (Proposition 3.1). On the other hand, we show a positive result for sequences related to the interpolation problem for analytic functions (Theorem 3.2).

Let us recall that the space $\mathscr{A}(\omega), \omega \subseteq \mathbb{R}^{d}$ an open subset, of real analytic functions $f: \omega \longrightarrow \mathbb{C}$ is equipped with the topology of the projective limit $\operatorname{proj}_{N} H\left(K_{N}\right)$, where $\left(K_{N}\right)$ is an exhaustion of $\omega$ by a sequence of compact sets

$$
K_{1} \subset \subset K_{2} \subset \subset \ldots \subset \subset K_{N} \subset \subset \ldots \subset \omega, \bigcup_{N \in \mathbb{N}} K_{N}=\omega,
$$

and $H(K)$ denotes the space of germs of analytic functions over $K$ with its natural LB-space topology. It is known (see [16, Prop. 1.7, 1.2]) that this topology is equal to the inductive limit topology ind $H(U)$, where $U$ runs over all open neighborhoods of $\omega$ in $\mathbb{C}^{d}$ and $H(U)$ denotes the Fréchet space of holomorphic functions on $U$ with the compact open topology. Thus $\mathscr{A}(\omega)$ is a complete, separable, ultrabornological, reflexive, webbed nuclear space with the approximation property (but non-metrizable and without a basis [7]) with the dual being a complete LF-space $\mathscr{A}(\omega)_{\beta}^{\prime}=\operatorname{ind} H\left(K_{N}\right)_{\beta}^{\prime}$. The space $\mathscr{A}(\omega)$ is the projective limit of a sequence of LB-spaces. Spaces of this type are called PLB-spaces. For more information on the space of real analytic functions, see [2], 4], [5, 7], 8] and [16].

For smooth functions on $\omega \subseteq \mathbb{R}^{d}$ we use the standard multi-index notation. So if $\alpha=\left(\alpha_{1}, \ldots, \alpha_{d}\right) \in \mathbb{N}^{d}$, then

$$
\frac{\partial^{|\alpha|}}{\partial x^{\alpha}} f=\frac{\partial^{|\alpha|}}{\partial x_{1}^{\alpha_{1}} \ldots \partial x_{d}^{\alpha_{d}}} f, \quad \delta_{z}(f):=f(z), \quad \delta_{z}^{\alpha}(f):=(-1)^{|\alpha|} \frac{\partial^{|\alpha|}}{\partial x^{\alpha}} f(z),
$$

where $|\alpha|=\alpha_{1}+\ldots+\alpha_{d}$.

The elements $\mu \in H\left(\mathbb{C}^{d}\right)^{\prime}$ are called analytic functionals. A compact subset $K \subset \mathbb{C}^{d}$ is called a carrier for $\mu$ if for every neighborhood $U \subset \subset \mathbb{C}^{d}$ of $K$ there is a continuity estimate

$$
|\mu(f)| \leq C \sup _{z \in U}|f(z)|
$$

We may identify $\mathscr{A}\left(\mathbb{R}^{d}\right)^{\prime}$ with the analytic functionals that are carried by some $K \subset \mathbb{R}^{d}$. In this case there is a smallest real carrier which is called the support of $\mu$ and denoted by $\operatorname{supp} \mu$ (see [19, p. $44 \mathrm{ff}$ ]). For open $\omega \subset \mathbb{R}^{d}$ we may identify $\mathscr{A}(\omega)^{\prime}$ with the analytic functionals $\mu$ with $\operatorname{supp} \mu \subset \omega$.

The other notation is standard. We refer for functional analysis to [17] and for complex analysis to [13]. For analytic functionals see [19] or [15]. 


\section{Distinguished OPEN SETS}

We will need some auxiliary notions. We call a nonnegative $C^{\infty}$-function $\psi$ : $\mathbb{R}^{d} \longrightarrow \mathbb{R}$ distinguished whenever it vanishes at infinity and

$$
\sup _{x \in \mathbb{R}^{d}}\left\|H_{\psi}(x)\right\|<1
$$

where $H_{\psi}$ denotes the Hessian of $\psi$ and $\|\cdot\|$ is the norm in the space of quadratic forms on the $d$-dimensional euclidean space. For every distinguished function $\psi$ we define

$$
\Omega^{\psi}:=\left\{z=x+i y \in \mathbb{C}^{d}:|y|^{2}<\psi(x)\right\}
$$

and call it a distinguished open set with basis $\omega:=\Omega^{\psi} \cap \mathbb{R}^{d}$. These sets and the construction below are variations of the proof of the Cartan-Grauert Theorem as given in [3, Prop. 1] and [10, Prop. 6 and Prop. 7].

Lemma 1.1. (a) Every distinguished open set $\Omega^{\psi}$ is a domain of holomorphy and for every $\alpha>0, \Omega_{\alpha}^{\psi}:=\left\{z:|y|^{2}<\psi(x)-\alpha\right\} \subset \subset \Omega^{\psi}$. Moreover, polynomials are dense in $H\left(\Omega^{\psi}\right)$ equipped with the compact-open topology.

(b) For every pair of open sets $U \subset W \subset \mathbb{C}^{d}, U$ distinguished, there is a distinguished set $\Omega^{\psi}$ with the basis $W \cap \mathbb{R}^{d}$ such that $U \subseteq \Omega^{\psi} \subseteq W$. If $U \subset \subset W$, then we can choose $\psi$ such that $U \subset \subset \Omega^{\psi}$.

(c) Every open set $\omega \subset \mathbb{R}^{d}$ has a basis of open neighbourhoods in $\mathbb{C}^{d}$ consisting of distinguished sets.

(d) Every compact set in $\mathbb{C}^{d}$ is contained in some bounded distinguished set.

Parts (a) and (c) imply a corollary going back to Cartan (see [11, Cor. II. 3.15]):

Corollary 1.2. Every open set $\omega$ in $\mathbb{R}^{d}$ has a basis of neighbourhoods in $\mathbb{C}^{d}$ consisting of domains of holomorphy.

Proof of Lemma 1.1. (a): Let $f(z):=|y|^{2}-\psi(x)$ for $z=x+i y \in \mathbb{C}^{d}$. Since $\left\|H_{\psi}(x)\right\|<1$, the Levi form of $f$ is positive definite. Thus, $f$ is plurisubharmonic on $\mathbb{C}^{d}$. Clearly $\Omega^{\psi}=\{z: f(z)<0\}$. Since $\psi$ vanishes at infinity, $\Omega_{\alpha}^{\psi} \subset \subset \Omega^{\psi}$. By [13, Cor. 5.4.3], every function holomorphic on a neighbourhood of $\overline{\Omega_{\alpha}^{\psi}}$ can be approximated uniformly on this set by entire functions.

(b): Let $U=\Omega^{\psi_{0}}$ and

$$
s(x):=\inf \left\{|w|: x+i(y+w) \notin W \text { for some } y,|y|^{2} \leq \psi_{0}(x)\right\} .
$$

Observe that

(i) since $\psi_{0}$ is continuous and $W$ is open, $s$ is lower semi-continuous;

(ii) $V:=\left\{x \in W \cap \mathbb{R}^{d}: s(x)>0\right\}$ is open and contains $(W \backslash U) \cap \mathbb{R}^{d}$;

(iii) if $U \subset \subset W$, then $V=W \cap \mathbb{R}^{d}$.

We choose a covering of $V$ by sets

$$
U_{r_{j}}\left(x_{j}\right)=\left\{x \in \mathbb{R}^{d}:\left|x-x_{j}\right|<r_{j}\right\} \subset \subset W \cap \mathbb{R}^{d} .
$$

We set

$$
\varphi(x)=\left\{\begin{array}{ll}
e^{\frac{1}{|x|^{2}-1}} & \text { for }|x|<1, \\
0 & \text { for }|x| \geq 1,
\end{array} \quad \psi_{1}(x)=\sum_{j=1}^{\infty} \varepsilon_{j} \varphi\left(\frac{1}{r_{j}}\left(x-x_{j}\right)\right) .\right.
$$


By (i) and a suitable choice of $\left(\varepsilon_{j}\right)$, we get that the series is convergent and

(iv) $\psi_{1}(x)<s^{2}(x)$;

(v) $\sup _{x \in \mathbb{R}^{d}}\left\|H_{\psi_{1}}(x)\right\|<1-\sup _{x \in \mathbb{R}^{d}}\left\|H_{\psi_{0}}(x)\right\|$.

By (v), $\psi:=\psi_{0}+\psi_{1}$ is a distinguished function. It is immediate that $U \subseteq \Omega^{\psi}$ and, by (iv), we get $\Omega^{\psi} \subseteq W$. By (ii), the basis of $\psi$ is equal to $W \cap \mathbb{R}^{d}$. Finally, if $U \subset \subset W$, then, by (iii), $\left.\psi_{1}\right|_{U \cap \mathbb{R}^{d}}>\delta>0$. Thus $U \subset \Omega_{\delta}^{\psi} \subset \subset \Omega$.

(c): Take in (b) $U=\emptyset$ and $\omega=W \cap \mathbb{R}^{d}$.

(d): It suffices to observe that if $\varphi$ is defined as in (b) and $\varphi_{a}:=a \varphi\left(\frac{x}{a}\right)$, then $H_{\varphi_{a}}=a^{-1} H_{\varphi}$. Therefore, for $a$ big enough, $\varphi_{a}$ is a distinguished function but $\varphi_{a} \longrightarrow+\infty$ uniformly on compact sets as $a \longrightarrow \infty$.

The proof of the following lemma follows the line of, e.g., the proof of [19] Théorème 111].

Lemma 1.3. Let $\omega$ be open and bounded in $\mathbb{R}^{d}, \Omega^{\psi}$ a distinguished set with basis $\omega$. Then for every $\mu \in H\left(\Omega^{\psi}\right)^{\prime} \cap \mathscr{A}\left(\mathbb{R}^{d}\right)^{\prime}$ we have $\operatorname{supp} \mu \subset \bar{\omega}$.

Proof. Let $\Omega \supset \bar{\omega}$ be open and bounded in $\mathbb{C}^{d}$. Let $\Omega_{1}$ be an open neighbourhood of $\mathbb{R}^{d}$ in $\mathbb{C}^{d}$ such that $\Omega^{\psi} \cap \Omega_{1} \subseteq \Omega$. By Lemma 1.1, there is a distinguished set $\Omega^{\psi_{1}}$ with basis $\mathbb{R}^{d}$ such that $\Omega^{\bar{\psi}} \subseteq \Omega^{\psi_{1}} \subseteq \Omega^{\psi} \cup \Omega_{1}$. Since $\Omega^{\psi_{1}}$ is a domain of holomorphy with covering $\Omega_{1} \cap \Omega^{\psi_{1}}$ and $\Omega^{\psi} \cap \Omega^{\psi_{1}}=\Omega^{\psi}$, the solution of the first Cousin problem yields the following exact sequence:

$0 \longrightarrow H\left(\Omega^{\psi_{1}}\right) \stackrel{j}{\longrightarrow} H\left(\Omega_{1} \cap \Omega^{\psi_{1}}\right) \oplus H\left(\Omega^{\psi}\right) \stackrel{\delta}{\longrightarrow} H\left(\Omega_{1} \cap \Omega^{\psi}\right) \longrightarrow 0$

where $j(f)=(f, f)$ and $\delta(f, g)=f-g$.

On $H\left(\Omega_{1} \cap \Omega^{\psi_{1}}\right) \oplus H\left(\Omega^{\psi}\right)$ we define $u(f, g)=\mu f-\mu g$. Since $u \circ j=0$ on $H\left(\mathbb{C}^{d}\right)$ and, by density of $H\left(\mathbb{C}^{d}\right)$ in $H\left(\Omega^{\psi_{1}}\right)$ (see Lemma 1.1 (a)), we have $u \circ j=0$ on $H\left(\Omega^{\psi_{1}}\right)$ and $u$ gives rise to an element $\mu \in H\left(\Omega_{1} \cap \Omega^{\psi}\right)^{\prime}$ that clearly extends the given $\mu \in \mathscr{A}\left(\mathbb{R}^{d}\right)^{\prime}$.

Since $\Omega^{\psi} \cap \Omega_{1} \subseteq \Omega$ this proves the result.

\section{Eidelheit SEQUenCES}

It turns out that a condition very similar to Eidelheit's characterization of Eidelheit sequences on Fréchet spaces is also necessary for any PLB-space.

Lemma 2.1. If $\left(f_{n}\right)_{n \in \mathbb{N}}$ is an Eidelheit sequence on a PLB-space $X=\operatorname{proj}_{N \in \mathbb{N}} X_{N}$, then for every $N \in \mathbb{N}$ we have

$$
\operatorname{dim}\left(\operatorname{span}\left\{f_{n}: n \in \mathbb{N}\right\} \cap X_{N}^{\prime}\right)<\infty .
$$

Proof. Let us assume that for some $N$ the condition (3) does not hold. Since $X_{N}$ is an LB-space, there is a bounded set $B \subset\left(X_{N}\right)_{\beta}^{\prime}$ such that $\operatorname{span}\left\{f_{n}: n \in \mathbb{N}\right\} \cap B$ has infinite dimension. Clearly, for a continuous surjection $T: X \longrightarrow \mathbb{C}^{\mathbb{N}}, T(g):=$ $\left(f_{n}(g)\right)_{n \in \mathbb{N}}$

$$
\begin{aligned}
\left(T^{\prime}\right)^{-1}(B) & \subseteq\left(T^{\prime}\right)^{-1}\left(B^{\circ \circ}\right)=\left\{g \in\left(\mathbb{C}^{\mathbb{N}}\right)^{\prime}: T^{\prime} g \in B^{\circ \circ}\right\} \\
& =\left\{g \in\left(\mathbb{C}^{\mathbb{N}}\right)^{\prime}:|g(T x)| \leq 1 \text { for } x \in B^{\circ}\right\}=\left(T\left(B^{\circ}\right)\right)^{\circ} .
\end{aligned}
$$

Since $T$ is open (as a surjective continuous operator from the webbed space onto a Fréchet space), $T\left(B^{\circ}\right)$ is a 0-neighbourhood and $\left(T\left(B^{\circ}\right)\right)^{\circ}$ is bounded in $\varphi=\left(\mathbb{C}^{\mathbb{N}}\right)^{\prime}$. 
Therefore $\left(T\left(B^{\circ}\right)\right)^{\circ}$ and $\left(T^{\prime}\right)^{-1}(B)$ are finite dimensional. Since $T^{\prime}$ is injective we get

$$
\operatorname{dim}\left(\operatorname{Im} T^{\prime} \cap B\right)=\operatorname{dim}\left(\operatorname{span}\left\{f_{n}: n \in \mathbb{N}\right\} \cap B\right)<\infty, \quad \text { a contradiction. }
$$

Remark. The condition (2) does not characterize Eidelheit sequences on an arbitrary PLB-space. In [6, Ex. 2.8] the authors constructed an example of a PLB-space $X$ with nuclear steps such that its ultrabornological associated topology makes it a nuclear LB-space. Clearly, no sequence on $X$ is Eidelheit (since it must be an Eidelheit sequence on its ultrabornological associated space, and therefore, on an LB-space). On the other hand, by the construction, $X$ cannot be represented as a projective limit of LB-spaces with surjective linking maps, and therefore, there exists a sequence $\left(f_{n}\right)_{n} \in \mathbb{N} \subseteq X^{\prime}$ satisfying (2).

Surprisingly, for the space $\mathscr{A}(\omega)$ the above condition is also sufficient.

Theorem 2.2. Let $\omega \subseteq \mathbb{R}^{d}$ be an arbitrary domain. Then there exists a domain of holomorphy $\Omega$ in $\mathbb{C}^{\bar{d}}\left(\Omega=\mathbb{C}^{d}\right.$ if $\left.\omega=\mathbb{R}^{d}\right), \Omega \cap \mathbb{R}^{d}=\omega$, such that for any sequence $\left(\mu_{n}\right)_{n \in \mathbb{N}} \subseteq \mathscr{A}(\omega)^{\prime}$ of linearly independent analytic functionals the following assertions are equivalent:

(a) $\left(\mu_{n}\right)_{n \in \mathbb{N}}$ is an Eidelheit sequence on $\mathscr{A}(\omega)$;

(b) $\left(\mu_{n}\right)_{n \in \mathbb{N}}$ is an Eidelheit sequence on $H(\Omega)$;

(c) for every compact set $K \subset \subset \omega$ we have $\operatorname{dim}\left(\operatorname{span}\left\{\mu_{n}: n \in \mathbb{N}\right\} \cap\left\{\mu \in \mathscr{A}\left(\mathbb{R}^{d}\right)^{\prime}: \operatorname{supp} \mu \subset K\right\}\right)<\infty$.

Proof. Clearly (b) $\Longrightarrow(\mathrm{a}) \Longrightarrow(\mathrm{c})$ follows from Lemma 2.1 . Observe that by [19, Lemma 112], $\mu$ extends to $\mu \in H(K)^{\prime}$ if and only if $\operatorname{supp} \mu \subseteq K$.

$(\mathrm{c}) \Longrightarrow(\mathrm{b})$ : Let $\left(\omega_{n}\right)$ be an exhaustion of $\omega, \omega_{n} \subset \subset \omega_{n+1}$ for $n \in \mathbb{N}$. By Lemma 1.1 (b), there exists an increasing family of distinguished sets $\Omega^{\psi_{n}}$ with bases $\omega_{n}$, $\Omega^{\psi_{n}} \subset \subset \Omega^{\psi_{n+1}}$ for $n \in \mathbb{N}$. In the case of $\omega=\mathbb{R}^{d}$ we can take $\Omega^{\psi_{n}}$ containing the ball of radius $n$ in $\mathbb{C}^{d}$ (use Lemma 1.1 (d)). We define $\Omega:=\bigcup_{n \in \mathbb{N}} \Omega^{\psi_{n}}$, which is a domain of holomorphy by Lemma 1.1 (a) and [13, 3.3.7]. By Lemma 1.3, the assumption implies that for every compact set $L \subset \subset \Omega$ we have

$$
\operatorname{dim}\left(\operatorname{span}\left\{\mu_{n}: n \in \mathbb{N}\right\} \cap\left\{\mu \in H(\Omega)^{\prime}: \operatorname{supp} \mu \subset L\right\}\right)<\infty .
$$

Therefore the classical Eidelheit theorem (see [9], [17, Th. 26.27]) yields the result.

\section{Factorization of Eidelheit sequences}

Proposition 3.1. For every Eidelheit sequence $\left(f_{n}\right)_{n} \in \mathbb{N}$ on $\mathscr{A}(\omega)$ there is an Eidelheit sequence $\left(g_{n}\right)_{n} \in \mathbb{N}$ on $\mathscr{A}(\omega)$ such that there is no operator $T: \mathscr{A}(\omega) \rightarrow$ $\mathscr{A}(\omega)$ so that $g_{n} \circ T=f_{n}$ for all $n \in \mathbb{N}$.

Proof. By Theorem 2.2, there is a domain of holomorphy $\Omega \subseteq \mathbb{C}^{d}$ such that $\Omega \cap \mathbb{R}^{d}=$ $\omega$ and $\left(f_{n}\right)_{n} \in \mathbb{N}$ is an Eidelheit sequence on $H(\Omega)$ as well. By [8] (compare [4]), there is a Fréchet quotient $F$ of $\mathscr{A}(\omega)$ that is not a quojection. Since $H(\Omega)$ is quasinormable, by [21, Th. 2.4], there is an Eidelheit sequence $\left(h_{n}\right)_{n \in \mathbb{N}}$ on $F$ such that for no operator $S: H(\Omega) \rightarrow F$ the factorization $h_{n} \circ S=f_{n}$ holds.

Let $q: \mathscr{A}(\omega) \rightarrow F$ be the quotient map. Observe that $g_{n}:=h_{n} \circ q$ is the Eidelheit sequence we are looking for. Indeed, if $g_{n} \circ T=f_{n}$, then $h_{n} \circ q \circ T=f_{n}$, which contradicts the choice of $\left(h_{n}\right)_{n \in \mathbb{N}}$. 
We will consider now Eidelheit sequences of the form $\left(\delta_{z_{n}}^{\alpha}\right)_{n \in \mathbb{N},|\alpha| \leq k_{n}}$ on $\mathscr{A}(\omega)$, $\omega \subseteq \mathbb{R}^{d}$, where $\left(k_{n}\right)_{n \in \mathbb{N}}$ is an arbitrary sequence of natural numbers and $\left(z_{n}\right)$ is a discrete sequence. We call such sequences interpolation sequences.

Theorem 3.2. Let $\left(f_{n}^{(1)}\right) \subseteq \mathscr{A}\left(\omega_{1}\right)^{\prime}$ and $\left(f_{n}^{(2)}\right) \subseteq \mathscr{A}\left(\omega_{2}\right)^{\prime}$ be two arbitrary interpolation sequences. There is always an operator $T: \mathscr{A}\left(\omega_{1}\right) \longrightarrow \mathscr{A}\left(\omega_{2}\right)$ such that $T^{\prime}$ maps $\left(f_{n}^{(2)}\right)_{n \in \mathbb{N}}$ one-to-one onto $\left(f_{n}^{(1)}\right)_{n} \in \mathbb{N}$.

The proof of Theorem 3.2 follows immediately from Corollary 3.4 and Lemmas 3.5 and 3.6 below.

Lemma 3.3. Let $\omega_{1} \subseteq \mathbb{R}^{d}$ and $\omega_{2} \subseteq \mathbb{R}^{d}$ be open domains, and let $\left(z_{n}\right) \subseteq \omega_{1}$ and $\left(y_{n}\right) \subseteq \omega_{2}$ be discrete sequences. Let $\left(k_{n}\right)_{n} \in \mathbb{N}$ be a sequence of natural numbers. Then there is a real analytic map $\varphi: \omega_{1} \longrightarrow \omega_{2}$ such that for every $n \in \mathbb{N}, \varphi\left(z_{n}\right)=y_{n}$, the Jacobian matrix $\mathscr{J} \varphi$ of $\varphi$ at the points $z_{n}$ is just the unit matrix and all the partial derivatives of $\varphi$ at $z_{n}$ of rank strictly larger than one and less than or equal to $k_{n}$ are vanishing.

Corollary 3.4. Under the notation of the previous lemma, the composition operator $C_{\varphi}: \mathscr{A}\left(\omega_{2}\right) \longrightarrow \mathscr{A}\left(\omega_{1}\right), C_{\varphi}(f):=f \circ \varphi$, satisfies

$$
C_{\varphi}^{\prime}\left(\delta_{z_{n}}^{\alpha}\right)=\delta_{y_{n}}^{\alpha} \quad \text { for }|\alpha| \leq k_{n}, n \in \mathbb{N}
$$

Proof of Lemma 3.3. By [5, Lemma 4.2], there is a surjective real analytic map $\psi: \mathbb{R}^{d} \longrightarrow \omega_{2}$ of constant rank $d$. It is easily seen that for every $z \in \mathbb{R}^{d}$ the map $C_{\psi}^{\prime}$ $\operatorname{maps} \operatorname{span}\left\{\delta_{z}^{\alpha}:|\alpha| \leq k\right\} \subseteq \mathscr{A}\left(\mathbb{R}^{d}\right)^{\prime}$ onto $\operatorname{span}\left\{\delta_{\psi(z)}^{\alpha}:|\alpha| \leq k\right\} \subseteq \mathscr{A}\left(\omega_{2}\right)^{\prime}$. By the classical interpolation problem on domains of holomorphy and Cor. 1.2, we find $\eta: \omega_{1} \rightarrow \mathbb{R}^{d}$ such that $\varphi:=\psi \circ \eta$ is the map we are looking for.

Lemma 3.5. Let $\left(y_{n}\right),\left(x_{n}\right)$ be sequences in $\mathbb{R}^{d}$ tending to infinity. Let $(\alpha(n))$ and $(\beta(n))$ be sequences of multi-indices such that if $y_{n}=y_{k}$, then $\alpha(n) \neq \alpha(k)$. Then there is an operator $T: \mathscr{A}\left(\mathbb{R}^{d}\right) \longrightarrow \mathscr{A}\left(\mathbb{R}^{d}\right)$ such that

$$
\left(\frac{\partial^{\alpha(n)}}{\partial x^{\alpha(n)}} T f\right)\left(y_{n}\right)=\left(\frac{\partial^{\beta(n)}}{\partial x^{\beta(n)}} f\right)\left(x_{n}\right) \quad \text { for every } n \in \mathbb{N} .
$$

Proof. Let $\left(k_{n}\right)$ be an increasing sequence of natural numbers such that $k_{n} \geq$ $\max \left\{|\alpha(l)|: y_{l}=y_{n}\right\}$. Let us choose $\varphi_{n}: \mathbb{C}^{d} \rightarrow \mathbb{C}^{d}, \varphi_{n}\left(\mathbb{R}^{d}\right) \subseteq \mathbb{R}^{d}$, holomorphic such that

$\varphi_{n}\left(y_{k}\right)=\left\{\begin{array}{ll}x_{n} & \text { for } y_{k}=y_{n}, \\ 0 & \text { for } y_{k} \neq y_{n},\end{array} \quad\right.$ and $\quad\left(\frac{\partial^{\beta}}{\partial x^{\beta}} \varphi_{n}\right)\left(y_{k}\right)=0 \quad$ for $1 \leq|\beta| \leq k_{n}$.

By the solution of the classical interpolation problem for entire functions, such $\varphi_{n}$ exist and we may assume that $\varphi_{n} \rightarrow 0$ uniformly on compact sets. Analogously, we define functions $g_{n}: \mathbb{C}^{d} \rightarrow \mathbb{C}$ such that $\left(\frac{\partial^{\alpha(n)}}{\partial x^{\alpha(n)}} g_{n}\right)\left(y_{n}\right)=1$ and

$$
\left(\frac{\partial^{\beta}}{\partial x^{\beta}} g_{n}\right)\left(y_{k}\right)=0 \quad \text { for }|\beta| \leq k_{n} \text { and either } y_{k} \neq y_{n} \text { or } y_{k}=y_{n}, \beta \neq \alpha(n) .
$$

Since the map

$$
R: H\left(\mathbb{C}^{d}\right) \rightarrow \mathbb{C}^{\mathbb{N}}, \quad R(f):=\left(\left(\frac{\partial^{\beta}}{\partial x^{\beta}} f\right)\left(y_{n}\right)\right)_{n \in \mathbb{N},|\beta| \leq k_{n}}
$$


is surjective and open, we may choose $g_{n}$ such that for every compact set $K \subseteq \mathbb{C}^{d}$,

$$
\sup _{z \in K} \sum_{n \in \mathbb{N}}\left|g_{n}(z)\right| n^{k_{n}+1}<+\infty \text {. }
$$

We can define the map

$$
T: \mathscr{A}\left(\mathbb{R}^{d}\right) \rightarrow \mathscr{A}\left(\mathbb{R}^{d}\right), \quad(T f)(z):=\sum_{n \in \mathbb{N}} g_{n}(z) \cdot\left(\frac{\partial^{\beta(n)}}{\partial x^{\beta(n)}} f\right)\left(\varphi_{n}(z)\right) .
$$

Clearly, the series converges for every $f \in \mathscr{A}\left(\mathbb{R}^{d}\right)$ and, by the Closed Graph Theorem, the map is continuous. Moreover, for each $n$,

$$
\begin{aligned}
\left(\frac{\partial^{\alpha(n)}}{\partial x^{\alpha(n)}} T f\right)\left(y_{n}\right) & =\sum_{y_{k}=y_{n}} \frac{\partial^{\alpha(n)}}{\partial x^{\alpha(n)}}\left[g_{k} \cdot\left(\frac{\partial^{\beta(k)}}{\partial x^{\beta(k)}} f\right) \circ \varphi_{k}\right]\left(y_{k}\right) \\
& =\sum_{y_{k}=y_{n}}\left(\frac{\partial^{\beta(k)}}{\partial x^{\beta(k)}} f\right)\left(x_{n}\right) \cdot\left(\frac{\partial^{\alpha(n)}}{\partial x^{\alpha(n)}} g_{k}\right)\left(y_{k}\right) \\
& =\frac{\partial^{\beta(n)}}{\partial x^{\beta(n)}} f\left(x_{n}\right) .
\end{aligned}
$$

This completes the proof.

Lemma 3.6. Let $\mathbb{R}$ be embedded in a standard way into $\mathbb{R}^{d}$, and let $\left(z_{n}\right)$ be a discrete sequence in $\mathbb{R}$. Then there are operators $T: \mathscr{A}(\mathbb{R}) \longrightarrow \mathscr{A}\left(\mathbb{R}^{d}\right)$ and $S: \mathscr{A}\left(\mathbb{R}^{d}\right) \longrightarrow \mathscr{A}(\mathbb{R})$ such that $T^{\prime}\left(\delta_{z_{n}}\right)=\left(\delta_{z_{n}}\right)$ and $S^{\prime}\left(\delta_{z_{n}}\right)=\left(\delta_{z_{n}}\right)$ for $n \in \mathbb{N}$.

Proof. One takes $T(f)\left(x_{1}, \ldots, x_{d}\right):=f\left(x_{1}\right), S(f)(x):=f(x, 0, \ldots, 0)$.

\section{REFERENCES}

[1] S. Banach, Théorie des Opérations Linéaires, Monografie Matematyczne, vol. 1, Warszawa, 1932.

[2] J. Bonet, P. Domański, D. Vogt, Interpolation of vector valued real analytic functions, J. London Math. Soc. 66 (2002), 407-420. MR 2003h:46056

[3] H. Cartan, Variétés analytiques réelles et variétés analytiques complexes, Bull. Soc. Math. France 85 (1957), 77-99. MR 20:1339]

[4] P. Domański, L. Frerick, D. Vogt, Fréchet quotients of spaces of real analytic functions, Studia Math. 159 (2003), 229-245.

[5] P. Domański, M. Langenbruch, Composition operators on spaces of real analytic functions, Math. Nach. 254-255 (2003), 68-86. MR 2004c:46036

[6] P. Domański, D. Vogt, A splitting theory for the space of distributions, Studia Math. 140 (2000), 57-77. MR 2001e:46125

[7] - The space of real analytic functions has no basis, Studia Math. 142 (2000), 187-200. MR 2001m:46044

[8] _ A non-trivial Fréchet quotient of the space of real analytic functions, Arch. Math. 81 (2003), 208-214.

[9] M. Eidelheit, Zur Theorie der Systeme linearer Gleichungen, Studia Math. 6 (1936), 139-148.

[10] H. Grauert, On Levi's problem and the imbedding of real analytic manifolds, Ann. of Math. 68 (1958), 460-472. MR 20:5299

[11] F. Guaraldo, P. Macri, A. Tancredi, Topics on Real Analytic Spaces, Vieweg, Braunschweig, 1986. MR 90j:32001

[12] G. Köthe, Topological Vector Spaces, vols. I and II, Springer, Berlin, 1969. MR 40:1750

[13] S. Krantz, Function Theory of Several Complex Variables, Wiley, New York, 1982. MR 84c:32001

[14] M. Langenbruch, Analytic extension of smooth functions, Results Math. 36 (1999), 281-296. MR 2000i:46026 
[15] A. Martineau, Sur les fonctionelles analytiques et la transformation de Fourier-Borel, J. Analyse Math. 11 (1963), 1-164. MR 28:2437

[16] Sur la topologie des espaces de fonctions holomorphes, Math. Ann. 163 (1966), 62-88. MR 32:8109

[17] R. Meise, D. Vogt, Introduction to Functional Analysis, Clarendon Press, Oxford, 1997. MR 98g:46001

[18] B. S. Mityagin, Approximative dimension and bases in nuclear spaces, Uspekhi Mat. Nauk 16 (1961), 63-132 (Russian); English transl.: Russian Math. Surveys 16 (1961), 59-128. MR 27:2837

[19] P. Schapira, Théorie des Hyperfunctions, Lecture Notes in Mathematics 126, Springer-Verlag, Berlin-Heidelberg-New York, 1970 MR 58:30195]

[20] D. Vogt, Kernels of Eidelheit matrices and related topics, Proc. Intern. Symp. Funct. Anal., Silivri 1985, Doga Tr. J. 10 (1986), 232-256. MR 88i:46020

[21] _ On two problems of Mityagin, Math. Nachr. 141 (1989), 13-25. MR 90h:46018

[22] _ Kernels of power series matrices, Trudy Math. Inst. Steklov 203 (1995), 377-381; Proc. Steklov Inst. Math. 3 (1995), 377-381. MR 97d:46003

[23] K. Yosida, Functional Analysis, Springer, Berlin, 1974. MR 50:2851

Faculty of Mathematics and Computer Science, A. Mickiewicz University Poznań and Institute of Mathematics, Polish Academy of Sciences (Poznań branch), ul. UmulTOWSKa 87, 61-614 Poznań, Poland

E-mail address: domanski@amu.edu.p1

Bergische Universität Wuppertal, FB Mathematik, Gaussstr. 20, D-42097 WupperTal, Germany

E-mail address: dvogt@math.uni-wuppertal.de 